\title{
Instrumentos para avaliação da farmacoterapia do idoso: uma revisão
}

Tools for assessing the pharmacotherapy of the elderly: a review

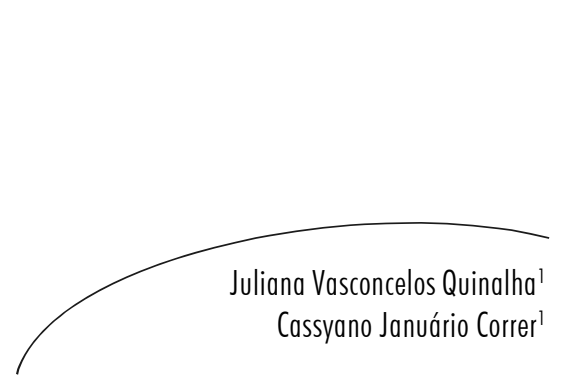

O envelhecimento é definido, no contexto cronológico, como sendo a partir de 60 anos nos países em desenvolvimento como o Brasil e a partir de 65 anos nos países desenvolvidos. Estudos mostram que, em termos absolutos, o Brasil será o $6^{\circ}$ país com a maior população idosa do mundo até o ano de 2025. Este dado se torna preocupante quando se percebe que os profissionais da área da saúde não estão preparados para atender essa demanda. São os idosos as pessoas que possuem maior número de diagnósticos e de uso de medicamentos; assim, a possibilidade do surgimento de problemas relacionados aos medicamentos é superior quando comparada à de pessoas jovens. Portanto, há necessidade de profissionais com conhecimento sobre as alterações fisiológicas e farmacológicas que ocorrem nos idosos, bem como sobre o uso de medicamentos por este grupo, a fim de avaliar a terapia prescrita e, quando necessário, sugerir alterações para otimização do tratamento. O farmacêutico pode ser este profissional. Este artigo traz uma revisão de alguns instrumentos de avaliação da farmacoterapia do idoso e os disponibiliza para que os profissionais possam utilizá-los durante o processo de acompanhamento destes pacientes.

\section{Abstract}

Aging is defined, in chronological context, as being over 60 years in developing countries like Brazil and over 65 in developed countries. Studies show that, in absolute terms, Brazil will become the 6th country with the largest elderly population in the world by 2025. This finding is worrying when you realize that health professionals are not prepared to meet this demand. Elderly people are the ones who have higher number of diagnoses and use of drugs, so the possibility of the emergence of problems related to drugs is higher when compared to that of young people. Therefore, there is a need for professionals with knowledge about the physiological and pharmacological changes that occur in the elderly as well as on the use of medicines

\footnotetext{
Universidade Federal do Paraná. Departamento de Farmácia. Grupo de Pesquisa em Prática Farmacêutica. Curitiba, PR, Brasil.

Correspondência / Correspondence

Cassyano Januário Correr

Universidade Federal do Paraná. Departamento de Farmácia

Av. Pref. Lothário Meissner, 632 - Jardim Botânico

80210-170, Curitiba, PR, Brasil

E-mail: cassyano@ufpr.br
}

Palavras-chave: Geriatria. Farmacoterapia. Instrumentos de avaliação. 
by this group in order to evaluate the therapy prescribed and, when necessary, suggest changes to optimize the treatment. The pharmacist can be this professional. This paper presents a review of some tools for evaluation of pharmacotherapy of the elderly and provides them for professionals, that can use them while monitoring these patients.
Key words: Geriatrics. Drug therapy. Tools of assessment.

\section{INTRODUÇÃO}

O envelhecimento populacional ocorre quando a taxa da população considerada idosa (60 anos ou mais no Brasil) é maior do que a da população de jovens. Isso pode acontecer devido a reduções na fecundidade e na mortalidade. Como consequência, a distribuição na pirâmide etária modifica-se, aumentando a idade média e a participação dos idosos no total da população, o que implica maior frequência de internações, maior número de consultas e maior uso de medicamentos ${ }^{1}$. Dentre os países mais populosos do mundo, o Brasil é o que apresenta maior velocidade no processo de envelhecimento demográfico. De acordo com as projeções das Nações Unidas e do IBGE, até o ano de 2025, o Brasil será o sexto país com maior população de idosos do mundo ${ }^{1,2}$.

Vários fatores podem influenciar a segurança, efetividade e sucesso da terapia farmacológica. Entre eles encontram-se as alterações anatômicas e funcionais naturais do envelhecimento (processo este conhecido como senescência) e por afecções que acometem o indivíduo idoso (processo denominado de senilidade), assim como a presença de múltiplas doenças, a polifarmácia, aumento da suscetibilidade a reações adversas a medicamentos (RAM's), mudanças na farmacologia e problemas na adesão ao tratamento ${ }^{3,4}$. É importante estar atento ao que faz parte da senescência e o que faz parte da senilidade, para não iniciar um tratamento desnecessariamente ou deixar de tratar um problema de saúde que merece cuidado. Pelo fato de os idosos consumirem a maior parte da produção mundial de medicamentos (média de 2-5 medicamentos por idoso) $)^{5}$ e serem mais sensíveis aos efeitos de certas classes farmacológicas, foram desenvolvidos vários estudos que identificam quais são os medicamentos inadequados para as pessoas de idade avançada ou cujo uso deve ser avaliado 6 . Assim, algumas listas foram propostas por pesquisadores da França, Canadá e Estados Unidos. Estas não são consideradas absolutas, sendo necessário o estudo da farmacoepidemiologia de cada país para predizer quais os medicamentos considerados impróprios de acordo com o perfil de idosos e características de cada região.

O farmacêutico pode auxiliar nos problemas mais comuns que ocorrem no uso de medicamentos por pessoas idosas, identificando medicamentos inapropriados, presença de interações, duplicidades terapêuticas, reações adversas, usos inadequados, automedicação e doses erradas, assim como fornecer informações sobre as doenças e os medicamentos prescritos, de modo que a adesão do paciente ao tratamento possa melhorar. Com isso, o farmacêutico pode garantir que a terapia prescrita continuará sendo necessária, efetiva no alcance dos objetivos terapêuticos, e segura.

Existem vários instrumentos disponíveis no Brasil, úteis na avaliação de pacientes idosos ${ }^{7}$. A maioria dos instrumentos existentes, entretanto, está relacionada com a área médica, incluindo testes de fluência verbal, instrumentos de avaliação do estado nutricional, do estado mental, de limitação das atividades funcionais, problemas de memória, avaliação do equilíbrio e da marcha e escalas de depressão geriátrica. Poucos são os instrumentos encontrados especificamente sobre a farmacoterapia no idoso, sendo que, quando encontrados, a maior parte deles tem pouca ou nenhuma repercussão no Brasil.

O objetivo desta revisão é reunir os principais instrumentos de avaliação úteis para otimização da farmacoterapia em idosos, tanto no âmbito hospitalar quanto ambulatorial. Para tanto, foi realizada uma pesquisa bibliográfica em 
periódicos científicos como jornais e revistas, monografias, livros, obras de divulgação e de referência, em bibliotecas convencionais e diferentes bases de dados (Lilacs, Medline, Scielo e Scopus), utilizando os seguintes termos de busca combinados entre si: idosos, geriatria, farmacoterapia, instrumentos de avaliação, otimização da farmacoterapia, medicamentos inadequados. Os artigos foram avaliados segundo a possibilidade de inclusão do instrumento na prática farmacêutica hospitalar e/ou comunitária no atendimento de pacientes geriátricos. Foram excluídos os artigos não adquiridos na íntegra a partir das fontes de pesquisa citadas e aqueles cuja aplicação era voltada para a prática médica.

\section{INSTRUMENTOS PARA AVALIAÇÃO DA FARMACOTERAPIA EM IDOSOS}

Instrumento para as discrepâncias dos medicamentos (medication discrepance tool - MDT)

O termo "discrepância" é definido como a diferença existente entre a medicação prescrita pelo médico e aquela que o paciente efetivamente toma, baseado nos frascos de medicamentos e relatos do próprio paciente ${ }^{8}$. A definição de outros autores diz que discrepância é a falta de acordo entre diferentes regimes medicamentosos que pode identificar possíveis erros de medicação que possam ocorrer na transição entre hospital e casa? .

Os tipos de discrepâncias de medicação encontrados podem estar relacionados ao paciente e/ ou ao sistema de cuidado. Algumas classes de medicamentos são mais frequentemente prescritas e, por isso, podem ser responsáveis por $50 \%$ de todas as discrepâncias de medicação ocorridas no cenário de transição hospital-casa. Entre elas, encontram-se: anticoagulantes (13\%); diuréticos (10\%); inibidores da enzima conversora de angiotensina [IECA] (10\%); agentes hipolipemiantes (10\%), e inibidores da bomba de prótons $(7 \%)^{9}$. O estudo realizado pela Universidade de Ciências da Saúde, Colorado, no ano de 2004 , teve como objetivo principal desenvolver um Instrumento para as Discrepâncias dos Medicamentos - MDT
(Anexo 1), capaz de auxiliar os profissionais de saúde na identificação e classificação dos problemas relacionados a medicamentos (PRM's), facilitando a resolução desses problemas e descrevendo ações apropriadas com relação ao paciente e ao sistema ${ }^{10}$.

A metodologia utilizada para o desenvolvimento de tal instrumento foi análise de 20 casos clínicos baseados em um grupo de pacientes com idade igual ou superior a 65 anos, que receberam alta hospitalar. As informações relevantes para identificação de discrepâncias foram obtidas através de visitas a casa dos pacientes e revisão da medicação, comparando os medicamentos que tomavam antes da hospitalização, prescrição de alta e medicamentos em uso após alta. Dois médicos clínicos gerais, dois farmacêuticos geriatras e duas enfermeiras avaliaram os casos para, desta forma, identificar quais itens incluir e como apresentá-los, bem como quais palavras utilizar para esclarecer as instruções a serem incluídas no MDT. Depois disso, foi realizado um teste de confiança para avaliar a capacidade de detecção de PRMs por parte dos profissionais de saúde e aplicabilidade dos itens, baseando-se nos resultados obtidos após uso desta ferramenta pelos profissionais convidados, os quais qualificaram o instrumento através do seu grau de concordância (baixo, bom, excelente). O estudo mostrou, através de análises estatísticas (Cohen), que os profissionais que usaram o MDT apresentaram boa capacidade para detectar problemas relacionados à medicação e que o teste serviu para distinguir os itens aplicáveis daqueles que não apresentaram aplicabilidade, quando então dois itens (dose e transporte incorretos) foram excluídos, obtendo assim a versão final do instrumento ${ }^{10}$.

A primeira parte do MDT é composta pelas causas e contribuição de fatores relacionados ao paciente e ao sistema de cuidado que podem levar à discrepância na medicação; a segunda parte contém as possíveis resoluções aplicáveis. A seção de discrepâncias relacionadas ao paciente tem como objetivo avaliar o paciente na gestão de seus medicamentos, diferenciando, por exemplo, a não adesão intencional daquela não intencional. A 
seção de discrepâncias relacionadas ao sistema tem como objetivo avaliar as práticas de trabalho no sistema de saúde e o regime efetivo da medicação para pacientes ambulatoriais. A seção final, "resoluções", possui como objetivo avaliar ações que possam ser tomadas para corrigir as discrepâncias encontradas ${ }^{10}$.

O MDT é um instrumento útil para identificar e caracterizar as discrepâncias dos medicamentos que possam acontecer com o paciente ambulatorial; entretanto, possui algumas limitações: nem todos os itens podem ser atribuídos exclusivamente a problemas de transição hospital-casa, mas podem ser considerados complementares àquilo que é específico da transição. Além disso, a prescrição de alta por si não é suficiente para determinar onde a discrepância ocorreu: muitas vezes é necessário que o médico complemente as informações. Para melhorar o MDT, o próprio autor sugere a inclusão de um item com os medicamentos utilizados sem prescrição médica ${ }^{10}$.

\section{Listas das medicações consideradas} inadequadas para o uso em idosos

Medicamento potencialmente inadequado para o uso em idosos é definido como qualquer medicamento cujos riscos são maiores que os benefícios. Alguns autores consideram o uso desses medicamentos como a maior causa de PRMs na terceira idade ${ }^{11}$, outros dizem ser responsável por inúmeras reações adversas; assim, é importante identificar quais são estes medicamentos, a fim de estabelecer uma terapia farmacológica adequada ${ }^{12}$.

O método Delphi, utilizado na elaboração das listas de medicações inadequadas, tem como objetivo consultar a opinião de um grupo de especialistas através de um questionário que circula repetidas vezes entre os respondentes, cujo anonimato é preservado. Primeiro, elabora-se um questionário, a partir da revisão da literatura, com informações suficientes para homogeneizar a linguagem e facilitar o raciocínio; em seguida, os convidados respondem individualmente às questões, podendo adicionar justificativas e opiniões. As respostas são analisadas estatisticamente, excluindo ou incluindo novos itens e as perguntas são repetidas no ciclo seguinte para que os respondentes reavaliem suas respostas, com base nos resultados e justificativas dadas pelo grupo na rodada anterior. Este processo é repetido várias vezes até que a divergência de opiniões esteja reduzida a um nível satisfatório, quando então se diz que foi obtido um consenso entre os especialistas ${ }^{13}$. Assim, com a resposta da última rodada, foram obtidos os instrumentos das medicações inadequadas para o uso em pessoas com 60 anos ou mais.

Todas as listas de medicações inadequadas para idosos apresentam como principal limitação uma rápida desatualização, pela constante retirada e inclusão de medicamentos no mercado farmacêutico e, além disso, a mais utilizada de todas (Critério de Beers) não sugere alternativas terapêuticas mais seguras para os idosos, como o fazem as mais recentes listas desenvolvidas em 1997 no Canadá e em 2007 na França ${ }^{12}$. Também, não são feitos estudos envolvendo eficácia, doses apropriadas, possíveis resultados negativos e sua probabilidade de ocorrência, associados à prescrição de medicamentos inadequados. Apesar de não identificar todos os medicamentos inadequados ou as consequências de seu uso, essas listas reúnem os principais casos encontrados na prática clínica e servem como guia para os profissionais da saúde. Porém, ser inadequado não significa que se trata de uma contraindicação absoluta; é preciso considerar a relação risco/ benefício para cada paciente, devido às possíveis comorbidades, estado funcional, prognósticos e medicamentos em uso.

As listas de medicamentos considerados inadequados para o uso em idosos descritas neste artigo poderão ser encontradas na íntegra na base de dados PUBMED.

\section{Critérios de Beers}

O Critério de Beers é o método mais utilizado para avaliar as características, com relação aos efeitos, dos medicamentos prescritos aos idosos. 
Este método foi primeiramente desenvolvido em 1991, baseado no estudo de idosos institucionalizados nos Estados Unidos ${ }^{11,14}$. Houve atualizações posteriores em 1997 para torná-lo mais aplicável, incluindo no estudo os idosos não institucionalizados, e mais recentemente em 2002, para incluir novas informações, avaliar potenciais efeitos adversos e incluir novos medicamentos cujo uso não é indicado em pacientes idosos ${ }^{15}$.

A primeira lista desenvolvida por Beers et al. ${ }^{14}$ em 1991 era composta de 19 medicamentos inadequados e 11 medicamentos cuja dose, frequência de uso e duração do tratamento eram inadequadas para pessoas com 65 anos ou mais. A lista atualizada em 1997 constava de 28 medicamentos a serem evitados devido à sua inadequação e 35 medicamentos inadequados para 15 condições patológicas específicas ${ }^{16}$. A mais recente lista, atualizada em 2002, selecionou os medicamentos a serem incluídos através de uma revisão sistemática para depois enviar aos especialistas e obter um consenso. Ela consta de 48 medicamentos que devem ser evitados em pessoas idosas por serem inadequados e medicamentos não adequados para 20 condições patológicas específicas ${ }^{16,17}$.

No Brasil, em 2008, foi analisada a lista de medicamentos genéricos, publicada no Diário Oficial da União em 12 de julho de 2004, e comprovada a aplicabilidade do instrumento ao constatar a presença de $6,7 \%$ do total de medicamentos da lista, incluídos nos critérios de Beers-Fick para medicamentos inadequados em idosos. Entretanto, faz ressalvas ao apontar o uso comum de antitussígenos, cinarizina, diltiazem, piracetam, quinolonas, xantinas, cremes, pomadas e colírios, os quais não fazem parte do instrumento, mas necessitam de cautela na sua prescrição ${ }^{18}$.

Apesar de necessitar de periódicas atualizações para continuar sendo válido, este instrumento possui fácil aplicação e memorização, podendo ser utilizado em diversos idiomas. Sugere-se a possibilidade de considerar o uso de medicamentos fitoterápicos, gravidade da doença, diagnóstico e medidas não farmacológicas ${ }^{16,18}$.
Medicamentos inapropriados para uso em idosos: Lista Francesa

No ano de 2006 na França, de modo semelhante ao critério proposto por Beers et al. ${ }^{14}$ anos antes, foi realizado um consenso entre especialistas de várias áreas para estabelecer uma lista de medicamentos inadequados para idosos deste país.

Para tanto, cada critério do questionário foi avaliado segundo a escala Likert de 1 a 5 pontos, onde 1 significa "total acordo com a inadequação" e 5 "total desacordo com a inadequação". Os itens com escore 1 e 2 foram mantidos na lista, enquanto os itens 4 e 5 foram excluídos; os itens com escore 3 (opinião duvidosa) foram apresentados novamente aos especialistas em outro momento. Também foi estabelecido para qual idade essa lista teria aplicação. O questionário era composto de duas categorias: medicamentos que devem ser evitados em idosos por não serem efetivos ou induzirem algum tipo de risco e medicamentos que devem ser utilizados apenas em condições médicas específicas ${ }^{12}$.

A lista resultante identifica 34 medicamentos de prescrição inadequada para pessoas com mais de 75 anos, pois nesta idade as mudanças farmacocinéticas e farmacodinâmicas são mais significativas para alterar a resposta dos medicamentos do que em idades mais jovens. Entre os medicamentos encontrados, 25 foram considerados impróprios devido à relação risco/ benefício desfavorável, um medicamento teve sua eficácia considerada questionável e oito com relação risco/benefício desfavorável e eficácia questionável ${ }^{12}$.

De todos os medicamentos ou classes de medicamentos apresentados na lista francesa, apenas alguns fazem parte da lista proposta por Beers, pois foram excluídos aqueles não disponíveis na França (pentazocina, trimetobenzamida, flurazepam, meperidina, orfenadrina, guanadrel, isoxsurpina, doxazosina, tioridazina, mesoridazina, ácido etacrínico e tireóide dessecada) ou considerados prejudiciais e não efetivos (todos os barbitúricos, excluindo 
apenas o fenobarbital). Diferentemente da lista de Beers, na lista proposta para a França não são utilizados estrógenos sem associação com progesterona para a menopausa, fenilbutazona foi incluída por induzir efeitos hematolíticos, fluoxetina não foi considerada maior indutora de problemas que qualquer outro inibidor $\mathrm{da}$ recaptação de serotonina e amiodarona foi considerada efetiva e capaz de induzir efeitos adversos como outros antiarrítmicos. Esta lista adiciona, além de vários medicamentos propostos por Beers, o uso de dois ou mais antiinflamatórios não esteroidais, dois ou mais medicamentos psicotrópicos da mesma classe terapêutica e alguns outros medicamentos com propriedades anticolinérgicas ${ }^{12}$.

\section{Medicamentos inapropriados para o uso em idosos: Lista Canadense}

Esse estudo foi desenvolvido através do método Delphi modificado, no Centro Médico Acadêmico no Canadá, em 1997, por McLeod et al. ${ }^{17}$, e discorda em relação a alguns medicamentos considerados por Beers inapropriados para o uso em pessoas idosas. É o caso dos anti-hipertensivos reserpina e clortalidona, da amitriptilina e do hipoglicemiante oral clorpropamida. Os autores também criticam a lista de Beers pela inclusão de medicamentos que estão em desuso, como ciclandelato, propoxifeno e isoxsuprina ${ }^{19}$.

Inicialmente, desenvolveu-se uma lista com 38 práticas inadequadas, classificadas nas categorias de medicamentos contraindicados devido à relação risco-benefício, medicamentos que podem causar interações com outros medicamentos e/ou interações com doenças e, posteriormente, essa lista foi enviada aos membros da equipe do consenso que julgaram as prescrições segundo as seguintes alternativas: possibilidade de aumento do risco de efeitos adversos, disponibilidade de terapias alternativas com efetividade igual ou superior e menor risco e, probabilidade da redução dessa prática conseguir diminuir a morbidade entre pessoas idosas. Com isso, foi possível gerar outra lista, agora com 71 práticas de prescrição de medicamentos para idosos, em que a condição clínica era descrita e uma terapia alternativa era sugerida para o caso ${ }^{19}$.

Para a avaliação da significância clínica das prescrições, os respondentes utilizaram uma escala de 1 a 4 ( 1 = sem significância e 4 = altamente significante) e emitiram seu parecer sobre a terapia alternativa proposta. A média de significância clínica variou de 2,53 a 3,83 e a variação da proporção de acordo ou desacordo com a terapia sugerida foi pequena ${ }^{19}$.

Neste instrumento encontram-se apenas as prescrições classificadas com média de significância clínica igual ou maior que 3,0, organizadas de acordo com a prescrição de medicamentos para o tratamento de doenças cardiovasculares, prescrição de medicamentos psicotrópicos, prescrição de AINES e outros analgésicos e prescrição de diferentes medicações. Das 38 práticas descritas no instrumento, 18 envolvem medicamentos contraindicados para pessoas idosas devido à desfavorável relação risco-benefício, 16 envolvem interações medicamento-doença e quatro envolvem interações medicamento-medicamento, sendo estas considerações as principais diferenças quando comparadas com a lista desenvolvida por Beers ${ }^{19}$.

Índice de adequação da medicação

(medication appropriateness index - MAl)

Para prescrever um medicamento de forma apropriada, o médico deve entender sobre a patologia diagnosticada, a farmacologia do medicamento a ser prescrito, assim como deve considerar as particularidades da fisiologia e farmacologia do organismo que irá receber o medicamento. Caso a prescrição não esteja adequada, esta poderá conter medicamentos desnecessários, não efetivos, prejudiciais, inviáveis e/ou muito caros, que geralmente contribuem para maior morbidade, internações e despesa para os pacientes. Como forma de garantir a qualidade das prescrições medicamentosas, desenvolveu-se um instrumento que avalia os elementos de uma prescrição e é aplicável para vários medicamentos, cenários e condições clínicas. Este instrumento, chamado de Índice de Adequação da Medicação 
- MAI (Anexo 2), foi elaborado por um farmacêutico clínico e um médico geriátrico, com base na revisão da literatura (MEDLINE) sobre medidas de avaliação ou escalas de avaliação da medicação $\mathrm{O}^{20} \mathrm{e}$, posteriormente, foi validado por Samsa et al. ${ }^{21}$, anos mais tarde.

$\mathrm{O}$ artigo que traz este instrumento, publicado por Joseph Hanlon et al. ${ }^{12}$ em 1992, primeiramente avaliou as prescrições medicamentosas de 10 pacientes idosos ambulatoriais, selecionados aleatoriamente, que recebiam cinco ou mais medicamentos e eram regularmente atendidos numa clínica de medicina interna nos Estados Unidos, para verificação da confiabilidade do MAI. Depois, dois farmacêuticos com especialização em geriatria selecionaram outra amostra de 10 pacientes geriátricos ambulatoriais, atendidos na mesma clínica médica e que utilizavam cinco ou mais medicamentos regularmente para verificação da generalização do instrumento. Para análise dos resultados, foram utilizados cálculos estatísticos de confiabilidade interclasse e intraclasse (kappa), de modo que o medicamento era considerado como completamente adequado combinando as 10 classificações e completamente inadequado se um ou mais itens recebessem classificação " 3 " ${ }^{20}$.

Neste instrumento, a prescrição é avaliada quanto a possíveis problemas relacionados aos medicamentos segundo 10 critérios essenciais: indicação (sinal, sintoma, doença ou condição para prescrição), efetividade (produção de resultado benéfico), dose (total de medicamentos tomados em 24 horas), administração (instruções para uso correto de um medicamento), comodidade/ praticidade (capacidade de ser usado ou colocado em prática), interações medicamentomedicamento (efeito que a administração de um medicamento tem em outro, geralmente prejudicial), interação medicamento-doença (efeito que um medicamento tem em uma doença ou condição pré-existente, geralmente prejudicial), duplicidade terapêutica (prescrição não benéfica ou arriscada de dois ou mais medicamentos de mesma classe farmacológica ou química), duração (período de tempo do tratamento) e custo (custo de um medicamento em comparação com outros agentes de igual eficácia e segurança). $\mathrm{O}$ índice segue uma escala de 3 pontos, onde 1 significa "uso apropriado do medicamento", 2 "uso limitadamente apropriado do medicamento", 3 "uso inapropriado do medicamento" e 9 "não sei”. Deste modo, a pontuação total por paciente se obtém pela soma da pontuação de cada um dos elementos analisados para cada medicamento, sendo que quanto mais alta a pontuação obtida na escala de 1 a 3, mais inapropriada a prescrição para o paciente idoso ${ }^{16,20,22}$. Muitas vezes, pode não ser possível avaliar determinados itens no momento da aplicação do instrumento, a exemplo do item efetividade; neste caso, o profissional deve assinalar o 9 "não sei”, a fim de não interferir no resultado, já que a avaliação é obtida apenas pelo somatório na escala de 1 a 3 pontos.

Trata-se de um método com instruções operacionais fáceis e úteis para revisar um grande número de medicamentos utilizados pelos pacientes idosos. Entretanto, possui algumas desvantagens, como a de não incluir em sua escala a presença de RAM (exceto aquelas provocadas por interações medicamento-medicamento e medicamento-doença), devido, segundo o autor, à existência de algoritmos próprios para isso; não incluir a presença de problema de saúde não tratado, qualidade de vida e não adesão ao tratamento; além de o tempo despendido para revisar cada medicamento ser de aproximadamente 10 minutos, o que pode impossibilitar sua aplicação em locais muito movimentados ${ }^{16,20,22}$.

\section{Instrumento para previsão de reações} adversas a medicamentos

Este instrumento foi elaborado a partir de um estudo envolvendo 186 idosos com idade igual ou superior a 60 anos, realizado pela enfermaria da Clínica Médica do Centro Hospitalar Municipal de Santo André, no período de 2002 a 2004. Foram excluídos do estudo os pacientes intubados, em coma ou já internados em outros setores do hospital, os quais possuíam condição clínica que não permitiria a coleta de dados para a pesquisa e identificação de Reações Adversas a 
Medicamentos (RAM), as quais foram classificadas conforme a gravidade e mecanismo. Os dados coletados se referiam a idade, sexo, escolaridade, história prévia de RAM ou de etilismo, diagnósticos, doses e uso de medicamentos inapropriados, avaliados conforme Critério de Beers, resultados do Mini-Exame do Estado Mental e da Escala de Atividades da Vida Diária, datas de internação, óbito e/ou alta, descrição de intercorrências ou complicação e exames complementares. Cada possível RAM identificada nesse estudo foi avaliada conforme seu grau de probabilidade, através do algoritmo de Naranjo ${ }^{23}$.

Do total dos pacientes avaliados $61,8 \%$ apresentaram pelo menos uma RAM no período em que estiveram internados, o que dá uma média de no mínimo uma e no máximo oito RAM para cada paciente, os quais apresentaram as seguintes características: idade entre 70-79 anos, maior número de diagnósticos e medicações prescritas, tempo de internação maior e uso dos seguintes medicamentos: furosemida, clortalidona, captopril, ciprofloxacino, eritromicina, insulina $\mathrm{NPH}$, glibenclamida, tenoxicam e diclofenaco ${ }^{23}$.

A partir do modelo de regressão logística, fixouse a possibilidade de ocorrência de RAM em $50 \%$ e elaborou-se o Instrumento de Previsão de RAM em Idosos (Anexo 3). A partir do número de diagnósticos, número de medicamentos e uso ou não de medicamentos inapropriados, é possível determinar o valor de corte da quantidade de medicamentos utilizados, de modo que, se for ultrapassado esse valor, o paciente apresentará probabilidade superior a $50 \%$ de desenvolver uma RAM. Por exemplo, se o paciente apresenta somente um diagnóstico e não usa nenhum medicamento inapropriado, só terá risco aumentado de RAM caso lhe seja prescrito número superior a 18 medicamentos; porém, se utilizar algum medicamento inadequado e apresentar mais de três diagnósticos, entrará para o grupo de maior risco à $R A M$, independentemente do número de medicamentos. A sensibilidade deste instrumento é de $88,7 \%$ e a especificidade de $40,8 \%{ }^{23}$.

Caso durante a avaliação seja identificado risco elevado de desenvolver RAM, o profissional de saúde deverá realizar o acompanhamento do paciente, reavaliando indicações e doses dos medicamentos, nível sérico (quando aplicável) e então decidir entre suspender a medicação, diminuir a dose ou associar algum outro medicamento ${ }^{23}$.

\section{CONCLUSÕES}

A revisão dos principais instrumentos de avaliação da farmacoterapia no idoso contidos neste artigo pode auxiliar os profissionais da saúde, inclusive o farmacêutico, pois considera os diversos medicamentos comumente prescritos e determinadas condições clínicas destes pacientes, além de acelerar o tempo para detecção de um PRM.

A contribuição deste artigo é demonstrar ao profissional farmacêutico as possíveis inadequações $\mathrm{da}$ farmacoterapia nos idosos e suas consequências do ponto vista do paciente, instigando o desejo de melhoria na qualidade do atendimento nas farmácias. A aplicabilidade dos instrumentos aqui propostos poderá ser avaliada em novos estudos e pelos farmacêuticos durante sua prática clínica, tanto na dispensação e aconselhamento dos pacientes, como na monitorização e avaliação dos resultados do tratamento. 


\section{REFERÊNCIAS}

1. Moreira MDM. Determinantes demográficos do envelhecimento brasileiro. 2000 [cited 200715 de nov.]; Available from: http:// www.abep.nepo.unicamp.br/docs/anais/pdf/ 2000/Todos/idot5_1.pdf

2. IBGE. Política do idoso no Brasil. 2000 [cited 200712 de out.]; Available from: http:// www.ibge.gov.br/home/presidencia/noticias/ 25072002pidoso.shtm

3. Jacob Filho W, Souza RR. Anatomia e fisiologia do envelhecimento. In: Atheneu, editor. Geriatria: Fundamentos, Clínica e Terapêutica. São Paulo; 2000. p. 31-40.

4. Williams CM. Using Medications Appropriately in Older Adults. American Family Physician. 2002; 66(10): 1917-24.

5. Castellar JI, Karnikowski MG, Vianna LG, Nobrega OT. [Study of the pharmacotherapy prescribed for older-adult in a Brazilian longterm care facility]. Acta Med Port. 2007 MarApr; 20(2): 97-105.

6. Nóbrega OT, Karnikowski MGO. A terapia medicamentosa no idoso: cuidados na medicação. 2005 [cited 200727 de nov.]; Available from: http://www.scielo.br/pdf/csc/ v10n2/a08v10n2.pdf

7. Andrade MA, Silva MVS, Freitas O. Assistência farmacêutica como estratégia para o uso racional de medicamentos em idosos. 2008 [cited $200821 \mathrm{de} \mathrm{fev.];} \mathrm{55-63].} \mathrm{Available} \mathrm{from:}$ http://www.uel.br/proppg/semina/pdf/ semina_25_1_20_17.pdf

8. Bedell SE, et al. Discrepancies in the use of medications: their extent and predictors in an outpatient practice. Arch Intern Med. $2000 \mathrm{Jul}$ 24; 160(14):2129-34.

9. Coleman EA, Smith JD, Raha D, Min SJ. Posthospital medication discrepancies: prevalence and contributing factors. Arch Intern Med. 2005 Sep 12; 165(16):1842-7.

10. Smith JD, Coleman EA, Min SJ. A new tool for identifying discrepancies in postacute medications for community-dwelling older adults. Am J Geriatr Pharmacother. 2004 Jun; 2(2):141-7.

11. Fu AZ, Jiang JZ, Reeves JH, Fincham JE, Liu GG, Perri M. Potentially inappropriate medication use and healthcare expenditures in the US community-dwelling elderly. 3rd. Med Care 2007 May; 45(5):472-6.
12. Laroche ML, Charmes JP, Merle L. Potentially inappropriate medications in the elderly: a French consensus panel list. Eur J Clin Pharmacol. 2007 Aug; 63(8):725-31.

13. Wright JTC, Giovinazzo RA. Delphi: uma Ferramenta de apoio ao planejamento prospectivo. 2000 [cited $200806 \mathrm{de} \mathrm{fev.];} \mathrm{Available}$ from: http://www.iea.usp.br/iea/tematicas/ futuro/projeto/delphi.pdf

14. Beers MH, Ouslander JG, Rollingher I, Reuben DB, Brooks J, Beck JC. Explicit criteria for determining inappropriate medication use in nursing home residents. UCLA Division of Geriatric Medicine. Arch Intern Med. 1991 Sep; 151(9):1825-32.

15. Petrone K, Katz P. Approaches to appropriate drug prescribing for the older adult. Prim Care. 2005 Sep; 32(3):755-75.

16. Ribeiro AQ, Araújo CMdC, Acurcio FDA, Magalhães SMS, Chaimowicz F. Qualidade do uso de medicamentos por idosos: uma revisão dos métodos de avaliação disponíveis. 2005 [cited 200713 de jun.]; Available from: http://www.scielo.br/ scielo.php?pid $=$ S141381232005000400026\&script $=$ sci_arttext

17. Fick DM, Cooper JW, Wade WE, Waller JL, Maclean JR, Beers MH. Updating the Beers criteria for potentially inappropriate medication use in older adults: results of a US consensus panel of experts. Arch Intern Med. 2003 Dec 822; 163(22): 2716-24.

18. Gorzoni ML, Fabbri RMA, Pires SL. Critérios de Beers-Fick e medicamentos genéricos no Brasil. Rev Assoc Med Bras. 2008; 54(4): 353-6.

19. McLeod PJ, Huang AR, Tamblyn RM, Gayton DC. Defining inappropriate practices in prescribing for elderly people: a national consensus panel. Cmaj. 1997 Feb 1; 156(3):385-91.

20. Hanlon JT, et al. A method for assessing drug therapy appropriateness. J Clin Epidemiol. 1992 Oct;45(10):1045-51.

21. Samsa GP, et al. A summated score for the medication appropriateness index: development and assessment of clinimetric properties including content validity. J Clin Epidemiol. 1994 aug; 47(8):891-6. 
22. Oscanoa T, Lira G. Calidad de prescripción de medicamentos en pacientes geriátricos. 2005 [cited 200727 de out.]; Available from: http://

www.scielo.org.pe/pdf/afm/v66n3/a02v66n3.pdf
23. Passarelli MCG, Jacob Filho W. Reações adversas a medicamentos em idosos: como prevê-las? Einstein. 2007; 5(3):24651.

Recebido: 04/8/2009

Aprovado: 02/6/2010 


\section{ANEXO 1}

\section{INSTRUMENTO PARA IDENTIFICAÇÃO DA DISCREPÂNCIA DA MEDICAÇÃO (MEDICATION DISCREPANCE TOOL - MDT)}

Esta ferramenta é projetada para facilitar a concordância do regime medicamentoso entre diversos âmbitos e prescritores

DESCRIÇÃO DA DISCREPÂNCIA ENCONTRADA (Completar um formulário para cada discrepância)

CAUSAS E CONTRIBUIÇÃO DE FATORES (Marque tudo que seja aplicável)

O texto em itálico sugere a perspectiva que o paciente tem e/ou intenção pretendida

Em relação ao paciente:

( ) Reação adversa a medicamento ou parte dos efeitos

( ) Intolerância

( ) A prescrição não foi atendida (o paciente não foi comprar ou não tinha o medicamento)

( ) A prescrição não foi necessária

( ) Dinheiro/barreiras financeiras

( ) Não adesão intencional

"Foi dito para eu tomar disso, mas en escolho não fazer isso"

( ) Não adesão não intencional (p. ex. falta de conhecimento)

"Eu não estou entendendo como tomar esse medicamento"

( ) Falta de habilidade em como usar o medicamento

"Talvez alguém tenha me mostrado, mas eu não consigo demonstrar para você que eu posso"

Em relação ao sistema:

( ) Prescrições com alergias /intolerâncias conhecidas

( ) Informação conflitante para diferentes fontes de informação;

Por exemplo, informações de alta indicando uma ação e dizer outra no frasco de comprimidos.

( ) Confusão entre nomes de marca e genérico

( ) Informação de alta incompleta/inexata/ilegível

Ou o paciente não é capaz de compreender a caligrafia ou a informação não é escrita em ordem.

( ) Duplicação; Tomar vários medicamentos com a mesma ação sem nenhum fundamento.

( ) Dosagem errada

( ) Quantidade errada

( ) Rótulo errado

( ) Deficiência cognitiva não diagnosticada

( ) Falta de cuidado/necessidade de assistência não identificada

( ) Limitações da visão/destreza não identificada

RESOLUÇÃO (marque tudo que seja aplicável)

( ) Recomendar parar de tomar/começar tomar/mudar o modo de utilização dos medicamentos

( ) Discutir benefícios potenciais e danos que a não adesão pode produzir

( ) Incentivar o paciente a ligar para um especialista ou clínico geral sobre o problema

( ) Incentivar o paciente a marcar consulta com um especialista ou clínico geral para discutir o problema

( ) Incentivar o paciente a falar com o farmacêutico sobre o problema

( ) Orientar o paciente (falta de conhecimento/habilidade para uso correto do medicamento)

( ) Providenciar recurso de informação para facilitar a adesão (ex. material impresso)

( ) Outros

Smith J. D, Coleman E. A, Min S. J., $2004{ }^{10}$. 


\section{ANEXO 2}

\begin{tabular}{|c|c|c|c|c|}
\hline ÍNDICE DE ADEQUAÇÃO DA MEDICAC & ÃO (MEDIC & & ATENESS INL & - MAI) \\
\hline Para avaliar se o medicamento é apropriado, fav & $\begin{array}{l}\text { responder a } \\
\text { aplicável: }\end{array}$ & & circular a pont & (escore) \\
\hline 1. Existe uma indicação para o medicamento? & 1 & 2 & 3 & 9 \\
\hline Comentários: & Indicado & & Não indicado & Não sei \\
\hline 2. O medicamento é efetivo para a condição? & 1 & 2 & 3 & 9 \\
\hline Comentários: & Efetivo & & Não efetivo & Não sei \\
\hline 3. A dosagem é correta? & 1 & 2 & 3 & 9 \\
\hline Comentários: & Correta & & Incorreta & Não sei \\
\hline 4. As admistrações são corretas? & 1 & 2 & 3 & 9 \\
\hline Comentários: & Correta & & Incorreta & Não sei \\
\hline 5. As admistrações são práticas? & 1 & 2 & 3 & 9 \\
\hline Comentários: & Prática & & Não práticas & Não sei \\
\hline 6. Existem interações medicamento- & $\underline{1}$ & 2 & 3 & 9 \\
\hline $\begin{array}{l}\text { medicamento clinicamente significantes? } \\
\text { Comentários: }\end{array}$ & Significante & & Insignificante & Não sei \\
\hline 7. Existem interações medicamento- doença/ & 1 & 2 & 3 & 9 \\
\hline $\begin{array}{l}\text { problema de saude clinicamente signiticantes? } \\
\text { Comentários: }\end{array}$ & Significante & & Insignificante & Não sei \\
\hline 8. Existe duplicação desnecessária com outros & 1 & 2 & 3 & 9 \\
\hline $\begin{array}{l}\text { medicamentos? } \\
\text { Comentários: }\end{array}$ & Necessária & & Desnecessária & Não sei \\
\hline 9. A duração do tratamento é aceitável? & 1 & 2 & 3 & 9 \\
\hline Comentários: & Aceitável & & Inaceitável & Não sei \\
\hline 10. Este medicamento é a alternativa de menor & 1 & 2 & 3 & 9 \\
\hline $\begin{array}{l}\text { custo em comparação a outros medicamentos } \\
\text { com a mesma utilidade? } \\
\text { Comentários: }\end{array}$ & Menos caro & & Mais caro & Não sei \\
\hline
\end{tabular}

INTERPRETAÇÃO: O índice segue uma escala de 3 pontos, onde 1 significa "uso apropriado do medicamento", 2 "uso limitadamente apropriado do medicamento", 3 "uso inapropriado do medicamento" e 9 "não sei". A pontuação total se obtém pela soma da pontuação de cada um dos elementos analisados para cada medicamento, sendo que quanto mais alta a pontuação na escala de 1 a 3 , mais inapropriada a prescrição para o paciente idoso.

Hanlon JT, Schmader KE, Samsa GP, Weinberger M, Uttech KM, Lewis IK, et al., $1992{ }^{20 .}$ 


\section{ANEXO 3}

\begin{tabular}{|c|c|c|}
\hline \multicolumn{2}{|c|}{ Instrumento de Previsão de RAM* em Idoso } \\
\hline & \multicolumn{2}{|c|}{ Uso de medicamento inapropriado } \\
\hline Número de diagnósticos & Não & Sim \\
\hline 1 & 18 & 9 \\
\hline 2 & 15 & 6 \\
\hline 3 & 11 & 0 \\
\hline 4 & 7 & 0 \\
\hline 5 & 4 & 2 \\
\hline
\end{tabular}

* RAM = reações adversas a medicamentos

INTERPRETAÇÃO: Se ultrapassar esses valores de corte obtidos, o paciente apresentará probabilidade superior a 0,5 de desenvolver uma RAM (possibilidade fixada de RAM=0,5). Exemplo, se o paciente apresenta um diagnóstico e não usa nenhum medicamento inapropriado, terá risco aumentado de RAM caso lhe seja prescrito número superior a 18 medicamentos; porém, se utilizar algum medicamento inadequado e apresentar mais de três diagnósticos entrará para o grupo de maior risco à RAM, independentemente do número de medicamentos.

Passarelli MCG, Jacob Filho W., $2007{ }^{23}$. 
